MIT-CTP 4004

\title{
A purely algebraic construction of a gauge and renormalization group invariant scalar glueball operator
}

\author{
D. Dudal $^{* a, b}$, S. P. Sorella ${ }^{\dagger c}$, N. Vandersicke ${ }^{\ddagger b}$, H. Verschelde Ves $^{\S b}$ \\ ${ }^{a}$ Center for Theoretical Physics, Massachusetts Institute of Technology, \\ 77 Massachusetts Avenue, Cambridge, MA 02139, USA \\ ${ }^{b}$ Ghent University, Department of Mathematical Physics and Astronomy \\ Krijgslaan 281-S9, 9000 Gent, Belgium \\ ${ }^{c}$ Departamento de Física Teórica, Instituto de Física, UERJ - Universidade do Estado do Rio de Janeiro \\ Rua São Francisco Xavier 524, 20550-013 Maracanã, Rio de Janeiro, Brasil
}

\begin{abstract}
This paper presents a complete algebraic proof of the renormalizability of the gauge invariant $d=4$ operator $F_{\mu v}^{2}(x)$ to all orders of perturbation theory in pure Yang-Mills gauge theory, whereby working in the Landau gauge. This renormalization is far from being trivial as mixing occurs with other $d=4$ gauge variant operators, which we identify explicitly. We determine the mixing matrix $Z$ to all orders in perturbation theory by using only algebraic arguments and consequently we can uncover a renormalization group invariant by using the anomalous dimension matrix $\Gamma$ derived from $Z$. We also present a future plan for calculating the mass of the lightest scalar glueball with the help of the framework we have set up.
\end{abstract}

\section{Introduction}

Quantum Chromodynamics (QCD) is the theory of the strong interactions which describes the force between quarks and gluons. At high energies, this theory is asymptotically free, while at low energies, only colorless bound states appear in nature due to confinement. So far, the mechanism of confinement is still poorly understood, since at low energies non-perturbative aspects play an important role. It is therefore of paramount importance to study objects which can bring us closer to the understanding of confinement. Glueballs are highly interesting candidates, as they are entirely composed of gluons, and therefore the gauge field itself is a crucial ingredient. For standard hadronic particles on the other hand, also matter fields are indispensable. Hence, glueballs have been widely investigated, experimentally, on the lattice and in various theoretical models [1].

So far, there is no clear experimental evidence for the existence of glueballs. If glueballs are observable particles, they would strongly mix with other states containing quarks. Due to this feature, a clear observation of a glueball state turns out to be rather difficult. However, there are already many indications for the existence of glueballs, and the debate is currently ongoing. It is worth mentioning here that several experiments are actually running and other ones are planned to start in the next future: PANDA [2], BES III [3] and GlueX [4] to name only a few. Glueballs might also play an important role in the quark gluon plasma, a case that will be studied at e.g. the heavy ion collision experiment ALICE at CERN [5].

\footnotetext{
david.dudal@ugent.be

sorella@uerj.br ; Work supported by FAPERJ, Fundação de Amparo à Pesquisa do Estado do Rio de Janeiro, under the program Cientista do Nosso Estado, E-26/100.615/2007.

‡ nele.vandersickel@ugent.be

§ henri.verschelde@ugent.be
} 
As no clear experimental data is yet available, the output of theoretical models ought to be compared with lattice data. In lattice gauge theories, there is no doubt about the existence of glueballs, although lattice calculations are still limited as they cannot determine the decay channels of glueballs. In contrast with possible experimental data, lattice calculations can however also consider pure gauge theory. A consensus on the lowest lying scalar glueball mass in the pure gauge gauge theory has already been reached : $M_{0^{++}} \sim 1.6 \mathrm{GeV}$ for $\mathrm{SU}(3)[6,7,8,9,10$, [1].

Many theoretical models have been investigated and compared with the lattice data. An extensive recent overview is given in [1]. Historically, the first model to describe glueballs is called the MIT bag model [12]. In this model, gluons are placed in a bag and confined by a boundary condition and a constant energy density $B$. This model, however, is rather phenomenological in nature. Other phenomenological models assume the gluons to have an effective mass [13, 14], which can be used to compose effective (potential) theories in which the masses of the different glueballs are calculated [15, 16, 17, 18].

A more direct way to deal with glueballs is by identifying suitable gauge invariant operators, which carry the correct quantum numbers to create/annihilate particular glueball states, and then calculating the corresponding correlators to get information on the mass. In particular, this route is followed in the widely used QCD sumrule approach [19, 20]. For example, the operator relevant for the lightest scalar glueball is $F^{2}(x) \equiv F_{\mu v}^{2}(x)$, hence the study of the correlator $\left\langle F^{2}(x) F^{2}(y)\right\rangle$. One takes into account perturbative as well as non-perturbative contributions, which are associated with condensates and instantons [19, 21]. Also in the AdS/QCD approach, glueball (correlators) have been investigated based on the assumption that there is an approximate dual gravity description [22, 23].

In the light of such correlator studies, it would be interesting to investigate the correlator $\left\langle F^{2}(x) F^{2}(y)\right\rangle$ within the Gribov-Zwanziger framework. The Gribov-Zwanziger action [24] was constructed in order to analytically implement the restriction to the Gribov region $\Omega$, defined as the set of field configurations fulfilling the Landau gauge condition and for which the Faddeev-Popov operator,

$$
\mathscr{M}^{a b}=-\partial_{\mu}\left(\partial_{\mu} \delta^{a b}+g f^{a c b} A_{\mu}^{c}\right)
$$

is strictly positive, namely

$$
\Omega \equiv\left\{A_{\mu}^{a}, \partial_{\mu} A_{\mu}^{a}=0, \mathcal{M}^{a b}>0\right\} .
$$

The boundary, $\partial \Omega$, of the region $\Omega$ is called the (first) Gribov horizon. This restriction is necessary to avoid the appearance of Gribov gauge copies in the Landau gauge [25]. Unfortunately, there are still a number of Gribov copies remaining, but the Gribov-Zwanziger action is so far the best approximation available. The Gribov-Zwanziger action is originally constructed as a non-local action. However, with the introduction of new fields, one can localize this action into the following form,

$$
\begin{aligned}
S_{0}= & S_{\mathrm{YM}}+\int \mathrm{d}^{4} x\left(b^{a} \partial_{\mu} A_{\mu}^{a}+\bar{c}^{a} \partial_{\mu} D_{\mu}^{a b} c^{b}\right)+\int \mathrm{d}^{4} x\left(\bar{\varphi}_{\mu}^{a c} \partial_{v} D_{v}^{a b} \varphi_{\mu}^{b c}-\bar{\omega}_{\mu}^{a c} \partial_{v} D_{v}^{a b} \omega_{\mu}^{b c}-g \partial_{v} \bar{\omega}_{\mu}^{a c} f^{a b m} D_{v}^{b e} c^{e} \varphi_{\mu}^{m c}\right) \\
& -\gamma^{2} g \int \mathrm{d}^{4} x\left(f^{a b c} A_{\mu}^{a} \varphi_{\mu}^{b c}+f^{a b c} A_{\mu}^{a} \bar{\varphi}_{\mu}^{b c}+\frac{4}{g}\left(N^{2}-1\right) \gamma^{2}\right)
\end{aligned}
$$

whereby $S_{\mathrm{YM}}$ denotes the Yang-Mills action, the fields $\left(\bar{\varphi}_{\mu}^{a c}, \varphi_{\mu}^{a c}\right)$ are a pair of complex conjugate bosonic fields and $\left(\bar{\omega}_{\mu}^{a c}, \omega_{\mu}^{a c}\right)$ are anticommuting fields needed to localize the original non-local action. The parameter $\gamma$ is fixed by a gap equation and implements the restriction to the Gribov region. This $\gamma$ introduces a mass scale into the theory which can consequently give rise to a nonvanishing pole in the glueball correlator. By taking into account the dynamics of the fields $(\bar{\varphi}, \varphi, \bar{\omega}, \omega)$, it became clear that a second mass scale $M^{2}$ emerges quite naturally, and this $M^{2}$ can also enter the glueball correlator expression. For more details on the Gribov-Zwanziger action, its renormalization and the dynamics of its constituent fields, we refer to [26, 27].

Once the operator $F_{\mu v}^{2}$ is introduced, the issues of renormalization and mixing complicate matters at the quantum level. If one wants to investigate $F_{\mu \nu}^{2}$ in a renormalizable setting, one should introduce this operator into the action by coupling it to a source $q$, and then renormalize that action. Therefore, before scrutinizing the more complicated Gribov-Zwanziger case, it is instructive to first completely investigate $F_{\mu \nu}^{2}$ within the usual Yang-Mills theory, quantized in the Landau gauge. Moreover, as $F_{\mu \nu}^{2}$ is not a renormalization group invariant, one could look for a renormalization group invariant operator, containing $F_{\mu v}^{2}$, since renormalization group invariance is beneficial when looking at physical quantities, in casu the glueball mass. This renormalization group invariant will turn out 
to coincide with the trace anomaly. However, we would like to avoid a direct use of the trace anomaly, as the renormalization of the trace anomaly itself, through that of the energy momentum tensor, is rather difficult and sometimes tricky [28]. Therefore, we shall focus on the direct renormalization of $F_{\mu v}^{2}$ in the Landau gauge, which also turns out to be far from trivial as a mixing with other (non gauge invariant) operators occurs. In 1974, [29] described the first attempt towards the renormalization of gauge invariant operators. In this paper, the renormalization of $F_{\mu v}^{2}$ at zero momentum was investigated to the first loop order, and the renormalization group invariant containing $F_{\mu v}^{2}$ determined. However, the paper [29] focused only on the integrated operatol 1$] \mathrm{d}^{4} x F_{\mu \nu}^{2}$. In addition, a generalization to the more complicated Gribov-Zwanziger case does not seem straightforward to implement in the language of [29]. Also, no clear proof of the higher order renormalization of the nonintegrated operator $F_{\mu v}^{2}(x)$ can be found. Notice that the passing from the integrated to the nonintegrated operator is not trivial, see [30], §12.6, and references in [31].

In [32, 33, 34], one has elaborated on the structure of the mixing matrix for the more general case of non-integrated gauge invariant operators, while in [35] a simplified proof of the renormalization of gauge invariant operators has been given from the perspective of the BRST cohomology of Yang-Mills gauge theories. In the light of this reference [35], the last paper published on these issues focusses in particular on some infrared subtleties [36]. One can appreciate the intrinsic difficulties arising when studying gauge invariant operators at the quantum level by noticing that in [37], results of [32] were used, while one of the authors of [37] quotes the same paper [32] again in later years in [36], mentioning that he finds the proof of [32] "very hard to understand".

Although the cohomological proof of [35] is of full generality, the results are of an abstract nature. In an oversimplifying nutshell, it was shown that each BRST invariant operator at the quantum level with ghost number zero, can be written as a strict gauge invariant operator 12 plus BRST exact piece, modulo terms that vanish upon using the equation of motions. For practical computations, it is however not sufficient to know which type of operators occur in the renormalization process, but also the explicit knowledge of all these operators is necessary.

For completeness, we also mention [38], concerning the renormalization of $F_{\mu v}^{2}$ in the background gauge formalism, which would however be of little use when looking at the Gribov-Zwanziger generalization.

Based on all the foregoing arguments, we have found it instructive to present in this paper a clean analysis of the renormalization of the operator $F_{\mu v}^{2}$ to all orders of perturbation theory. The proof shall be given in the framework of algebraic renormalization [39], and we shall retrieve a renormalization matrix, restricted by various Ward identities. Next to suitable adaptations of the usual Landau gauge Ward identities, we also identify a new powerful identity, relevant in the discussion of the renormalization matrix, which form shall be in perfect agreement with the argumentation given in [30, 36]. We stress that our analysis is purely algebraic, and in this sense differs from the argumentation given in [30, 36]. Moreover, we shall also be able to completely fix the mixing matrix to all orders and this without calculating any loop diagram. Multiple checks will be presented, which will confirm the results. For example, we shall recover in an independent fashion well-known nonrenormalization relations in the Landau gauge [39], here stemming from the renormalization analysis of $F_{\mu v}^{2}$.

In summary, in section II we shall give an overview of the 3 different classes of operators which can mix with $F_{\mu v}^{2}$ before going into the detailed algebraic renormalization of $F_{\mu v}^{2}$. In section III, the mixing matrix will be determined to all orders in perturbation theory and, armed with this result, we shall be able to construct a renormalization group invariant in section IV. We end this paper with a discussion in section V.

\section{Renormalization of the Yang-Mills action with inclusion of the operator $F_{\mu \nu}^{2}$}

\subsection{Introduction}

The most natural way to study the lightest scalar glueball is by determining the correlator $\left\langle F^{2}(x) F^{2}(y)\right\rangle$. This correlator can be obtained by adding the operator $F_{\mu \nu}^{2}$ to the ordinary Yang-Mills action by coupling it to a source $q(x)$.

Indeed, the action we start from reads,

$$
\Sigma_{\text {n.r. }}=\underbrace{\int \mathrm{d}^{4} x \frac{1}{4} F_{\mu \nu}^{2}}_{S_{\mathrm{YM}}}+\underbrace{\int \mathrm{d}^{4} x\left(b^{a} \partial_{\mu} A_{\mu}^{a}+\bar{c}^{a} \partial_{\mu} D_{\mu}^{a b} c^{b}\right)}_{S_{\mathrm{gf}}}+\int \mathrm{d}^{4} x \frac{q}{4} F_{\mu \nu}^{2},
$$

1. Or the operator at zero momentum.

2. This means containing only the field strength and covariant derivatives. 
whereby $S_{\mathrm{gf}}$ is the Landau gauge fixing part. For the benefit of the reader, let us already mention that this action is BRST invariant,

$$
s \Sigma_{\text {n.r. }}=0,
$$

with all the BRST transformations of the fields and the source given by

$$
\begin{array}{llrl}
s A_{\mu}^{a}=-\left(D_{\mu} c\right)^{a}, & s c^{a}=\frac{1}{2} g f^{a b c} c^{b} c^{c}, & \\
s \bar{c}^{a}=b^{a}, & s b^{a}=0, & s q=0,
\end{array}
$$

and $s$ nilpotent,

$$
s^{2}=0
$$

In this fashion, the correlator is given by

$$
\left[\frac{\delta}{\delta q(y)} \frac{\delta}{\delta q(x)} Z^{c}\right]_{q=0}=\left\langle F^{2}(x) F^{2}(y)\right\rangle
$$

with $Z^{c}$ the generator of connected Green functions. However, it will turn out that the action (2.1) is not renormalizable. Indeed, as the operator $F_{\mu v}^{2}$ has mass dimension 4, it could mix with other operators of the same dimension. The question arises which kind of extra operators we need to consider.

\subsection{3 classes of operators}

In general, we can distinguish between 3 different classes of dimension 4 operators. Firstly, the class $C_{1}$ contains all the truly gauge invariant operators. These are the BRST closed but not exact operators like $F_{\mu v}^{2}$. These are constructed from the field strength $F_{\mu \nu}^{a}$ and the covariant derivative $D_{\mu}^{a b}$. Secondly, the class $C_{2}$ consists of BRST exact operators, e.g. $s\left(\bar{c}^{a} \partial_{\mu} A_{\mu}^{a}\right)$. The third class $C_{3}$ contains operators which will vanish upon using the equations of motion, e.g. $A_{\mu}^{a} \frac{\delta S}{\delta A_{\mu}^{a}}$, with $S=S_{\mathrm{YM}}+S_{\mathrm{gf}}$.

Now, one can intuitively easily understand that these 3 different classes will mix in a certain way [30, 36]. Firstly, bare operators from the class $C_{2}$ cannot receive contributions from gauge invariant operators $\left(C_{1}\right)$. Indeed, taking the matrix element of a bare BRST exact operator from $C_{2}$ between physical states will give a vanishing result, if there would be a renormalized gauge invariant contribution from $C_{1}$ in its expansion, there would be a nonvanishing contribution, clearly a contradiction. Secondly, as a $C_{3}$ operator will vanish upon using the equations of motion, while a $C_{1}$ - and a $C_{2}$ operator in general do not, a $C_{3}$ operator cannot receive corrections from the $C_{1}$ and/or $C_{2}$ class.

Thus, the mixing matrix will have an upper triangular form,

$$
\left(\begin{array}{c}
\mathcal{F}_{0} \\
\mathcal{L}_{0} \\
\mathcal{H}_{0}
\end{array}\right)=\left(\begin{array}{ccc}
Z_{\mathcal{F} \mathcal{F}} & Z_{\mathcal{F} \mathcal{L}} & Z_{\mathcal{F} \mathcal{H}} \\
0 & Z_{\mathcal{L} \mathcal{L}} & Z_{\mathcal{L} \mathcal{H}} \\
0 & 0 & Z_{\mathcal{H} \mathcal{H}}
\end{array}\right)\left(\begin{array}{c}
\mathcal{F} \\
\mathcal{L} \\
\mathcal{H}
\end{array}\right),
$$

whereby $\mathcal{F}, \mathcal{L}, \mathcal{H}$ are operators belonging, respectively, to the $C_{1}, C_{2}$ and $C_{3}$ class.

We shall however not use these observations, and only rely on a formal algebraic analysis [39]. All constraints on e.g. the mixing matrix should be encoded in the Ward identities.

For further use, let us elaborate a bit more on the equation of motion like terms, using a scalar field for notational simplicity. A term $\sim \frac{\delta S}{\delta \varphi}$ shall give rise to contact terms when taking expectation values. Using partial path integration, one finds

$$
\begin{aligned}
\left\langle\varphi\left(x_{1}\right) \varphi\left(x_{2}\right) \ldots \varphi\left(x_{n+1}\right) \frac{\delta S}{\delta \varphi(y)}\right\rangle & =\int[\mathrm{d} \phi] \varphi\left(x_{1}\right) \varphi\left(x_{2}\right) \ldots \varphi\left(x_{n+1}\right) \frac{\delta S}{\delta \varphi(y)} \mathrm{e}^{-S} \\
& =-\int[\mathrm{d} \Phi] \varphi\left(x_{1}\right) \varphi\left(x_{2}\right) \ldots \varphi\left(x_{n+1}\right) \frac{\delta}{\delta \varphi(y)} \mathrm{e}^{-S} \\
& =\int[\mathrm{d} \Phi] \frac{\delta}{\delta \varphi(y)}\left[\varphi\left(x_{1}\right) \varphi\left(x_{2}\right) \ldots \varphi\left(x_{n+1}\right)\right] \mathrm{e}^{-S} \\
& =\sum_{k=1}^{n+1} \delta\left(x_{k}-y\right)\left\langle\varphi\left(x_{1}\right) \varphi\left(x_{2}\right) \ldots \varphi\left(x_{k-1}\right) \varphi\left(x_{k+1}\right) \ldots \varphi\left(x_{n+1}\right)\right\rangle .
\end{aligned}
$$


We used the symbolic notation $\int[\mathrm{d} \phi]$ for the integration over all the present fields. Introducing the $Z$-factors for the fields $\varphi$, one also learns that $\varphi(y) \frac{\delta S}{\delta \varphi(y)}$ does not need any renormalization factor, and thus that it is finite when introduced into correlators 3 . Moreover, if $x_{k} \neq y, k=1, \ldots, n$, the 1.h.s. of (2.7) will vanish as the r.h.s. does. On the other hand, it is easily recognized from (2.7) that the integrated operator $\int \mathrm{d}^{4} y \varphi(y) \frac{\delta S}{\delta \varphi(y)}$ is nothing more than a counting operator when inserted into a correlator, i.e.

$$
\left\langle\varphi\left(x_{1}\right) \varphi\left(x_{2}\right) \ldots \varphi\left(x_{n}\right) \int \mathrm{d}^{4} y \varphi(y) \frac{\delta S}{\delta \varphi(y)}\right\rangle=n\left\langle\varphi\left(x_{1}\right) \varphi\left(x_{2}\right) \ldots \varphi\left(x_{n}\right)\right\rangle .
$$

\subsection{The starting action}

We can now propose a more complete starting action than (2.1). Besides the gauge invariant operator $F_{\mu v}^{2}$ belonging to the first class $C_{1}$, we also introduce the BRST closed operator $s(\bar{\partial} c A) \equiv s\left(\partial_{\mu} \bar{c}^{a} A_{\mu}^{a}\right)$, coupled to a new dimensionless source $\eta$. As we want this new source to only enter the cohomological trivial part of the action, we shall introduce a BRST doublet $(\lambda, \eta)$,

$$
s \eta=\lambda
$$

and add the following term to the action (2.1),

$$
\int \mathrm{d}^{4} x s\left(\eta \bar{c}^{a} \partial_{\mu} A_{\mu}^{a}\right)=\int \mathrm{d}^{4} x\left(\lambda \partial_{\mu} \bar{c}^{a} A_{\mu}^{a}+\eta\left(\partial_{\mu} b^{a} A_{\mu}^{a}+\partial_{\mu} \bar{c}^{a} D_{\mu}^{a b} c^{b}\right)\right) .
$$

The BRST doublet-structure is highly useful in order construct the most general invariant counterterm [39].

Hence, the classical starting action is given by

$$
S_{\mathrm{cl}}=S_{\mathrm{YM}}+\int \mathrm{d}^{4} x\left(b^{a} \partial_{\mu} A_{\mu}^{a}+\bar{c}^{a} \partial_{\mu} D_{\mu}^{a b} c^{b}\right)+\int \mathrm{d}^{4} x q \underbrace{\frac{1}{4} F_{\mu \nu}^{2}}_{\mathcal{F}}+\int \mathrm{d}^{4} x \lambda \partial_{\mu} \bar{c}^{a} A_{\mu}^{a}+\int \mathrm{d}^{4} x \eta \underbrace{\left(\partial_{\mu} b^{a} A_{\mu}^{a}+\partial_{\mu} \bar{c}^{a} D_{\mu}^{a b} c^{b}\right)}_{\mathcal{L}} .
$$

Later in this paper, we shall also introduce the equation of motion terms from class $C_{3}$. Notice that in principle, also $s\left(\bar{c}^{a} \partial_{\mu} A_{\mu}^{a}\right)$ is another independent $d=4$ BRST exact operator which could play a role. It shall however turn out that the renormalization analysis closes without this operator, therefore we decided to immediately discard it.

We can now proceed with the study of this action, using the formalism of algebraic renormalization [39]. A first step is to introduce a term $S_{\text {ext }}$,

$$
S_{\mathrm{ext}}=\int \mathrm{d}^{4} x\left(-K_{\mu}^{a}\left(D_{\mu} c\right)^{a}+\frac{1}{2} g L^{a} f^{a b c} c^{b} c^{c}\right)
$$

needed to define the nonlinear BRST transformations of the gauge and ghost fields. $K_{\mu}^{a}$ and $L^{a}$ are two new sources, invariant under the BRST symmetry $s$. Therefore, the enlarged action is given by

$$
\begin{aligned}
\Sigma= & S_{\mathrm{YM}}+\int \mathrm{d}^{4} x\left(b^{a} \partial_{\mu} A_{\mu}^{a}+\bar{c}^{a} \partial_{\mu} D_{\mu}^{a b} c^{b}\right)+\int \mathrm{d}^{4} x\left(-K_{\mu}^{a}\left(D_{\mu} c\right)^{a}+\frac{1}{2} g L^{a} f^{a b c} c^{b} c^{c}\right) \\
& +\int \mathrm{d}^{4} x q \frac{1}{4} F_{\mu \nu}^{2}+\int \mathrm{d}^{4} x \lambda \partial_{\mu} \bar{c}^{a} A_{\mu}^{a}+\int \mathrm{d}^{4} x \eta\left(\partial_{\mu} b^{a} A_{\mu}^{a}+\partial_{\mu} \bar{c}^{a} D_{\mu}^{a b} c^{b}\right),
\end{aligned}
$$

and it will reduce itself to equation (2.11), once the sources $K_{\mu}^{a}$ and $L^{a}$ are set to zero at the end. Likewise, also $\lambda$ can be set to zero at that point.

A second step in the process of algebraic renormalization is to determine all the Ward identities obeyed by the action (2.13), which we have summarized here:

- $\quad$ The Slavnov-Taylor idenitity:

$$
\mathcal{S}(\Sigma)=\int \mathrm{d}^{4} x\left(\frac{\delta \Sigma}{\delta K_{\mu}^{a}} \frac{\delta \Sigma}{\delta A_{\mu}^{a}}+\frac{\delta \Sigma}{\delta L^{a}} \frac{\delta \Sigma}{\delta c^{a}}+b^{a} \frac{\delta \Sigma}{\delta \bar{c}^{a}}+\lambda \frac{\delta \Sigma}{\delta \eta}\right)=0 .
$$

3. The implied limit $x_{n+1} \rightarrow y$ might seem problematic due to the appearance of a $\delta(0)$ in the last term of the r.h.s. of 2.7]. However, $\delta(0)=0$ in dimensional regularization. 
- The Landau gauge condition:

$$
\frac{\delta \Sigma}{\delta b^{a}}=\partial_{\mu} A_{\mu}^{a}-\partial_{\mu}\left(\eta A_{\mu}^{a}\right)
$$

- $\quad$ The modified antighost equation:

$$
\frac{\delta \Sigma}{\delta \bar{c}^{a}}+\partial_{\mu} \frac{\delta \Sigma}{\delta K_{\mu}^{a}}-\left(\partial_{\mu} \eta\right) \frac{\delta \Sigma}{\delta K_{\mu}^{a}}=0
$$

- $\quad$ The ghost Ward identity:

$$
\int \mathrm{d}^{4} x\left(\frac{\delta}{\delta c^{a}}+g f^{a b c}\left(\bar{c}^{b} \frac{\delta}{\delta b^{c}}\right)\right) \Sigma=g \int \mathrm{d}^{4} x f^{a b c}\left(K_{\mu}^{b} A_{\mu}^{c}-L^{b} c^{c}\right) .
$$

The term $\Delta_{\mathrm{cl}}^{a}$, being linear in the quantum fields $A_{\mu}^{a}, c^{a}$, is a classical breaking.

- $\quad$ The extra integrated Ward identity:

$$
\int \mathrm{d}^{4} x\left(\frac{\delta \Sigma}{\delta \lambda}-\eta \frac{\delta \Sigma}{\delta \lambda}+\bar{c}^{a} \frac{\delta \Sigma}{\delta b^{a}}\right)=0 .
$$

Apart from some small adaptations, the first 5 symmetries are similar to the ones in the ordinary Yang-Mills action. Moreover, we also find an extra Ward identity w.r.t. the new doublet $(\lambda, \eta)$. This last identity will enable us to take into account in a purely algebraic way the effects related to the composite operators coupled to the sources $(\lambda, \eta)$. We underline here that this is the power of the algebraic formalism: by a well chosen set of sources to introduce the relevant operators, one can hope to find additional Ward identities which, in turn, will constrain the theory at the quantum level, including the characterization of the most general counterterm. As such, a good choice of sources can considerably simplify the renormalization analysis.

\subsection{The counterterm}

When we turn to the quantum level, we can use these symmetries to characterize the most general allowed invariant counterterm $\Sigma^{c}$. Following the algebraic renormalization procedure, $\Sigma^{c}$ is an integrated local polynomial in the fields and sources with dimension bounded by four, and with vanishing ghost number. The previous, nonanomalous, Ward identities imply the following constraints on $\Sigma^{c}$ :

- The linearized Slavnov-Taylor identity:

$$
\begin{gathered}
\mathcal{B}_{\Sigma} \Sigma^{c}=0, \quad \mathcal{B}_{\Sigma}^{2}=0 \\
\mathcal{B}_{\Sigma}=\int \mathrm{d}^{4} x\left(\frac{\delta \Sigma}{\delta K_{\mu}^{a}} \frac{\delta}{\delta A_{\mu}^{a}}+\frac{\delta \Sigma}{\delta A_{\mu}^{a}} \frac{\delta}{\delta K_{\mu}^{a}}+\frac{\delta \Sigma}{\delta L^{a}} \frac{\delta}{\delta c^{a}}+\frac{\delta \Sigma}{\delta c^{a}} \frac{\delta}{\delta L^{a}}+b^{a} \frac{\delta}{\delta \bar{c}^{a}}+\lambda \frac{\delta}{\delta \eta}\right) .
\end{gathered}
$$

- The Landau gauge condition:

$$
\frac{\delta \Sigma^{c}}{\delta b^{a}}=0
$$

- The modified antighost equation:

$$
\frac{\delta \Sigma^{c}}{\delta \bar{c}^{a}}+\partial_{\mu} \frac{\delta \Sigma^{c}}{\delta K_{\mu}^{a}}-\left(\partial_{\mu} \eta\right) \frac{\delta \Sigma}{\delta K_{\mu}^{a}}=0 .
$$

- $\quad$ The ghost Ward identity:

$$
\int \mathrm{d}^{4} x\left(\frac{\delta}{\delta c^{a}}+g f^{a b c}\left(\bar{c}^{b} \frac{\delta}{\delta b^{c}}\right)\right) \Sigma^{c}=0
$$




\begin{tabular}{|c|c|c|c|c|}
\hline & $A_{\mu}^{a}$ & $c^{a}$ & $\bar{c}^{a}$ & $b^{a}$ \\
\hline \hline dimension & 1 & 0 & 2 & 2 \\
\hline ghostnumber & 0 & 1 & -1 & 0 \\
\hline
\end{tabular}

Table 1: Quantum numbers of the fields.

\begin{tabular}{|c|c|c|c|c|c|}
\hline & $K_{\mu}^{a}$ & $L^{a}$ & $q$ & $\eta$ & $\lambda$ \\
\hline \hline dimension & 3 & 4 & 0 & 0 & 0 \\
\hline ghostnumber & -1 & -2 & 0 & 0 & 1 \\
\hline
\end{tabular}

Table 2: Quantum numbers of the sources.

- $\quad$ The extra integrated Ward identity:

$$
\int \mathrm{d}^{4} x\left(\frac{\delta \Sigma^{c}}{\delta \lambda}-\eta \frac{\delta \Sigma^{c}}{\delta \lambda}\right)=0
$$

To construct the most general counterterm, Tables 1 and 2 , listing the dimension and ghost number of the various fields and sources, are useful. There is however one subtlety concerning counterterms quadratic (or higher) in the sources. Only looking at the dimensionality, the ghost number and the constraints on the counterterm, it is a priori not forbidden to consider terms of the the form $\left(q^{2} \ldots\right),\left(\eta^{2} \ldots\right),(q \eta \ldots),\left(q^{3} \ldots\right)$, etc., i.e. terms of quadratic and higher order in the sources. If these terms are allowed, an infinite tower of counterterms would be generated and it would be impossible to prove the renormalizability of the action as new divergences are being generated, which cannot be absorbed in terms already present in the starting action. However, we can give a simple argument why we may omit this class of terms. Assume that we would also introduce the following term of order $q^{2}$ in the action,

$$
\sim \int \mathrm{d}^{4} x q^{2} \frac{F_{\mu v}^{2}}{4} .
$$

Subsequently, when calculating the correlator, this term would give rise to an extra contact term contribution,

$$
\left[\frac{\delta}{\delta q(z)} \frac{\delta}{\delta q(y)} \int[\mathrm{d} \phi] \mathrm{e}^{-S}\right]_{q=0}=\underbrace{\left\langle F^{2}(z) F^{2}(y)\right\rangle}_{\text {term due to part in } q}+\underbrace{\delta(y-z)\left\langle F^{2}(y)\right\rangle}_{\text {term due to part in } q^{2}} .
$$

As eventually we are only interested in the correlator for $z \neq y$, we can thus neglect the term (2.25). In fact, when looking at the case $z=y$, we should also couple a source to the novel composite operator $F^{4}$, which is not our current interest. We can repeat this kind of argument for all other terms of higher order in the sources.

There is one exception to the previous remark: we cannot neglect higher order terms of the type $(K q \ldots)$ and $(K \eta \ldots)$ due to the modified antighost equation,

$$
\frac{\delta \Sigma^{c}}{\delta \bar{c}^{a}}+\partial_{\mu} \frac{\delta \Sigma^{c}}{\delta K_{\mu}^{a}}-\left(\partial_{\mu} \eta\right) \frac{\delta \Sigma}{\delta K_{\mu}^{a}}=0 .
$$

The second term of this equation differentiates the counterterm w.r.t. the source $K_{\mu}^{a}$, while the first term w.r.t. the field $\bar{c}^{a}$. Therefore, for the construction of the counterterm fulfilling all the constraints, we still need to include terms of order $K q$ and $K \eta$, as when deriving w.r.t $K_{\mu}^{a}$, these terms will become of first order in the sources, just as the term $\propto \frac{\delta \Sigma^{c}}{\delta \bar{c}^{a}}$. However, at the end, after having completely characterized the counterterm, we can ignore this class of terms again.

We are now ready to construct the counterterm. Firstly, making use of general results on the cohomology of gauge theories [39], the most general integrated polynomial of dimension 4 in the fields and sources, with vanishing ghost number and which takes into account the previous remarks on the terms quadratic in the sources, can be written 


$$
\begin{aligned}
\Sigma^{c}= & a_{0} \int \mathrm{d}^{4} x \frac{1}{4} F_{\mu \nu}^{2}+b_{0} \int \mathrm{d}^{4} x \frac{q}{4} F_{\mu \nu}^{2}+\mathcal{B}_{\Sigma} \int \mathrm{d}^{4} x\left\{a_{1}\left(K_{\mu}^{a}+\partial_{\mu} \bar{c}^{a}\right) A_{\mu}^{a}+a_{2} L^{a} c^{a}+a_{3} b^{a} \bar{c}^{a}+a_{4} g f^{a b c} \bar{c}^{a} \bar{c}^{b} c^{c}\right\} \\
& +\mathcal{B}_{\Sigma} \int \mathrm{d}^{4} x\left\{b_{1} q\left(K_{\mu}^{a}+\partial_{\mu} \bar{c}^{a}\right) A_{\mu}^{a}+b_{2} q \bar{c}^{a} \partial_{\mu} A_{\mu}^{a}+b_{3} q b^{a} \bar{c}^{a}+b_{4} q g f^{a b c} \bar{c}^{a} \bar{c}^{b} c^{c}\right\} \\
& +\mathcal{B}_{\Sigma} \int \mathrm{d}^{4} x\left\{c_{1} \eta K_{\mu}^{a} A_{\mu}^{a}+c_{2} \eta \partial_{\mu} \bar{c}^{a} A_{\mu}^{a}+c_{3} \eta \bar{c}^{a} \partial_{\mu} A_{\mu}^{a}+c_{4} \eta b^{a} \bar{c}^{a}+c_{5} \eta g f^{a b c} \bar{c}^{a} \bar{c}^{b} c^{c}\right\} \\
& +\mathcal{B}_{\Sigma} \int \mathrm{d}^{4} x\left\{d_{1} \lambda \bar{c}^{a} \bar{c}^{a}\right\} .
\end{aligned}
$$

Secondly, we can simplify this counterterm by imposing all the constraints (2.19)-(2.24). After a certain amount of algebra, we eventually obtain

$$
\begin{aligned}
\Sigma^{c}= & a_{0} \int \mathrm{d}^{4} x \frac{1}{4} F_{\mu \nu}^{2}+b_{0} \int \mathrm{d}^{4} x \frac{q}{4} F_{\mu \nu}^{2}+a_{1} \int \mathrm{d}^{4} x\left(A_{\mu}^{a} \frac{\delta S_{\mathrm{YM}}}{\delta A_{\mu}^{a}}+A_{\mu}^{a} \frac{\delta \widehat{S}_{\mathrm{YM}}}{\delta A_{\mu}^{a}}+K_{\mu}^{a} \partial_{\mu} c^{a}+\partial_{\mu} \bar{c}^{a} \partial_{\mu} c^{a}-\eta \partial_{\mu} \bar{c}^{a} \partial_{\mu} c^{a}\right) \\
& +b_{1} \int \mathrm{d}^{4} x q\left(A_{\mu}^{a} \frac{\delta S_{\mathrm{YM}}}{\delta A_{\mu}^{a}}+K_{\mu}^{a} \partial_{\mu} c^{a}+\partial_{\mu} \bar{c}^{a} \partial_{\mu} c^{a}\right)
\end{aligned}
$$

with

$$
\widehat{S}_{\mathrm{YM}}=\frac{1}{4} \int \mathrm{d}^{4} x q F_{\mu v}^{2} .
$$

Now that we have constructed the most general counterterm obeying all the Ward identities, we can neglect, as previously described, the term in $K q$. Therefore, the final counterterm becomes,

$$
\begin{aligned}
\Sigma^{c}= & a_{0} \int \mathrm{d}^{4} x \frac{1}{4} F_{\mu \nu}^{2}+b_{0} \int \mathrm{d}^{4} x \frac{q}{4} F_{\mu \nu}^{2}+a_{1} \int \mathrm{d}^{4} x\left(A_{\mu}^{a} \frac{\delta S_{\mathrm{YM}}}{\delta A_{\mu}^{a}}+A_{\mu}^{a} \frac{\delta \widehat{S}_{\mathrm{YM}}}{\delta A_{\mu}^{a}}+K_{\mu}^{a} \partial_{\mu} c^{a}+\partial_{\mu} \bar{c}^{a} \partial_{\mu} c^{a}-\eta \partial_{\mu} \bar{c}^{a} \partial_{\mu} c^{a}\right) \\
& +b_{1} \int \mathrm{d}^{4} x q\left(A_{\mu}^{a} \frac{\delta S_{\mathrm{YM}}}{\delta A_{\mu}^{a}}+\partial_{\mu} \bar{c}^{a} \partial_{\mu} c^{a}\right) .
\end{aligned}
$$

\subsection{Introducing the equations of motion}

We still have to introduce the equations of motion as described in Section 2.2 as these can enter the operator $\mathcal{F}$. So far, we have found an action $\Sigma$ with corresponding counterterm $\Sigma^{c}$. Let us perform the linear shift on the gluon field $A_{\mu}^{a}$,

$$
A_{\mu}^{a} \rightarrow A_{\mu}^{a}+J A_{\mu}^{a},
$$

with $J(x)$ a novel local source. This way of introducing the relevant gluon equation of motion operator shall turn out to be very efficient, as it allows us to uncover the finiteness of this kind of operator. Indeed, this shift basically corresponds to a redefinition of the gluon field, and has to be consistently done in the starting action and counterterm. Performing the shift in the action gives rise to the following shifted action $\Sigma^{\prime}$,

$$
\begin{aligned}
\Sigma^{\prime}= & S_{\mathrm{YM}}+\int \mathrm{d}^{4} x\left(b^{a} \partial_{\mu} A_{\mu}^{a}+\bar{c}^{a} \partial_{\mu} D_{\mu}^{a b} c^{b}\right)+\int \mathrm{d}^{4} x\left(-K_{\mu}^{a}\left(D_{\mu} c\right)^{a}+\frac{1}{2} g L^{a} f^{a b c} c^{b} c^{c}\right) \\
& +\int \mathrm{d}^{4} x q \frac{1}{4} F_{\mu \nu}^{2}+\int \mathrm{d}^{4} x \lambda \partial_{\mu} \bar{c}^{a} A_{\mu}^{a}+\int \mathrm{d}^{4} x \eta\left(\partial_{\mu} b^{a} A_{\mu}^{a}+\partial_{\mu} \bar{c}^{a} D_{\mu}^{a b} c^{b}\right) \\
& +\int \mathrm{d}^{4} x J \underbrace{A_{\mu}^{a} \frac{\delta S_{\mathrm{YM}}}{\delta A_{\mu}^{a}}}_{\mathscr{H}}+\int \mathrm{d}^{4} x J\left\{-\partial_{\mu} b^{a} A_{\mu}^{a}+g f_{a k b} A_{\mu}^{k} c^{b} \partial_{\mu} \bar{c}^{a}\right\},
\end{aligned}
$$


where we see the relevant gluon equation of motion term, $\mathcal{H}$, emerging. Again, we have neglected higher order terms in the sources, as the argument (2.26) still holds. Analogously, we find a shifted counterterm,

$$
\begin{aligned}
\Sigma^{\prime c}= & a_{0} \int \mathrm{d}^{4} x \frac{1}{4} F_{\mu v}^{2}+b_{0} \int \mathrm{d}^{4} x \frac{q}{4} F_{\mu v}^{2}+a_{1} \int \mathrm{d}^{4} x\left(A_{\mu}^{a} \frac{\delta S_{\mathrm{YM}}}{\delta A_{\mu}^{a}}+A_{\mu}^{a} \frac{\delta \widehat{S}_{\mathrm{YM}}}{\delta A_{\mu}^{a}}+K_{\mu}^{a} \partial_{\mu} c^{a}+\partial_{\mu} \bar{c}^{a} \partial_{\mu} c^{a}-\eta \partial_{\mu} \bar{c}^{a} \partial_{\mu} c^{a}\right) \\
& +b_{1} \int \mathrm{d}^{4} x q\left(A_{\mu}^{a} \frac{\delta S_{\mathrm{YM}}}{\delta A_{\mu}^{a}}+\partial_{\mu} \bar{c}^{a} \partial_{\mu} c^{a}\right)+a_{0} \int \mathrm{d}^{4} x\left(J A_{\mu}^{a} \frac{\delta S_{\mathrm{YM}}}{\delta A_{\mu}^{a}}\right) \\
& +a_{1} \int \mathrm{d}^{4} x J\left(2 A_{\mu}^{a} \partial_{\mu} \partial_{v} A_{v}^{a}-2 A_{\mu}^{a} \partial^{2} A_{\mu}^{a}+9 g f_{a b c} A_{\mu}^{a} A_{v}^{b} \partial_{\mu} A_{v}^{c}+4 g^{2} f_{a b c} f_{c d e} A_{\mu}^{a} A_{v}^{b} A_{\mu}^{d} A_{v}^{e}\right)
\end{aligned}
$$

where one can neglect again the higher order terms in the sources.

One could also introduce the other similar equation of motion terms, by introducing linear shifts for the $b^{a}, c^{a}, \bar{c}^{a}$ fields. However, the corresponding equation of motion operators will not mix with $F_{\mu v}^{2}$ and are therefore unnecessary to establish the renormalizability of the action (2.33).

\subsection{Stability and the renormalization (mixing) matrix}

Finally, it remains to discuss the stability of the classical action, i.e. to check whether $\Sigma^{\prime c}$ can be reabsorbed in the classical action $\Sigma^{\prime}$ by means of a multiplicative renormalization of the coupling constant $g$, the fields $\{\phi=A, c, \bar{c}, b\}$ and the sources $\{\Phi=L, K, q, \eta, \lambda, J\}$, namely

$$
\Sigma^{\prime}(g, \phi, \Phi)+h \Sigma^{\prime c}=\Sigma\left(g_{0}, \phi_{0}, \Phi_{0}\right)+o\left(h^{2}\right),
$$

with $h$ the infinitesimal perturbation parameter. The bare fields, sources and parameters are defined as

$$
\begin{aligned}
K_{0 \mu}^{a} & =Z_{K} K_{\mu}^{a}, & A_{0 \mu}^{a}=Z_{A}^{1 / 2} A_{\mu}^{a}, & g_{0}=Z_{g} g, \\
L_{0}^{a} & =Z_{L} L^{a}, & c_{0}^{a}=Z_{c}^{1 / 2} c^{a}, & \\
q_{0} & =Z_{q} q, & \bar{c}_{0}^{a} & =Z_{\bar{c}}^{1 / 2} \bar{c}^{a}, \\
\eta_{0} & =Z_{\eta} \eta, & b_{0}^{a}=Z_{b}^{1 / 2} b^{a}, & \\
J_{0} & =Z_{J} J, & & \\
\lambda_{0} & =Z_{\lambda} \lambda . & &
\end{aligned}
$$

We also propose the following mixing matrix,

$$
\left(\begin{array}{c}
q_{0} \\
\eta_{0} \\
J_{0}
\end{array}\right)=\left(\begin{array}{ccc}
Z_{q q} & Z_{q \eta} & Z_{q J} \\
Z_{\eta q} & Z_{\eta \eta} & Z_{\eta J} \\
Z_{J q} & Z_{J \eta} & Z_{J J}
\end{array}\right)\left(\begin{array}{c}
q \\
\eta \\
J
\end{array}\right),
$$

which will represent the mixing of the operators $\mathcal{F}, \mathcal{L}$ and $\mathcal{H}$. If we try to absorb the counterterm into the original action, we ultimately find,

$$
\begin{aligned}
Z_{g} & =1-h \frac{a_{0}}{2}, \\
Z_{A}^{1 / 2} & =1+h\left(\frac{a_{0}}{2}+a_{1}\right),
\end{aligned}
$$

and

$$
\begin{aligned}
Z_{\bar{c}}^{1 / 2} & =Z_{c}^{1 / 2}=Z_{A}^{-1 / 4} Z_{g}^{-1 / 2}=1-h \frac{a_{1}}{2} \\
Z_{b} & =Z_{A}^{-1} \\
Z_{K} & =Z_{c}^{1 / 2} \\
Z_{L} & =Z_{A}^{1 / 2}
\end{aligned}
$$

results which are known from the renormalization of the original Yang-Mills action in the Landau gauge [39]. 
In addition, we also find the following mixing matrix

$$
\left(\begin{array}{ccc}
Z_{q q} & Z_{q \eta} & Z_{q J} \\
Z_{\eta q} & Z_{\eta \eta} & Z_{\eta J} \\
Z_{J q} & Z_{J \eta} & Z_{J J}
\end{array}\right)=\left(\begin{array}{ccc}
1+h\left(b_{0}-a_{0}\right) & 0 & 0 \\
h b_{1} & 1 & 0 \\
h b_{1} & 0 & 1
\end{array}\right),
$$

and for completeness, the $Z$-factor of $\lambda$ reads,

$$
Z_{\lambda}=Z_{c}^{-1 / 2} Z_{A}^{-1 / 2}
$$

as the counterterm does not contain the source $\lambda$.

Once having this mixing matrix at our disposal, we can of course pass to the corresponding bare operators. For this, we shall need the inverse of the mixing matrix (2.40),

$$
\left(\begin{array}{c}
q \\
\eta \\
J
\end{array}\right)=\left(\begin{array}{ccc}
\frac{1}{Z_{q q}} & 0 & 0 \\
-\frac{Z_{J q}}{Z_{q q}} & 1 & 0 \\
-\frac{Z_{J q}}{Z_{q q}} & 0 & 1
\end{array}\right)\left(\begin{array}{c}
q_{0} \\
\eta_{0} \\
J_{0}
\end{array}\right)
$$

Now we can determine the corresponding mixing matrix for the operators, since insertions of an operator correspond to derivatives w.r.t. to the appropriate source of the generating functional $Z^{c}(q, \eta, J)$. In particular,

$$
\begin{aligned}
\mathcal{F}_{0} & =\frac{\delta Z^{c}(q, \eta, J)}{\delta q_{0}}=\frac{\delta q}{\delta q_{0}} \frac{\delta Z^{c}(q, \eta, J)}{\delta q}+\frac{\delta \eta}{\delta q_{0}} \frac{\delta Z^{c}(q, \eta, J)}{\delta \eta}+\frac{\delta J}{\delta q_{0}} \frac{\delta Z^{c}(q, \eta, J)}{\delta J} \\
\Rightarrow \mathcal{F}_{0} & =\frac{1}{Z_{q q}} \mathcal{F}-\frac{Z_{J q}}{Z_{q q}} \mathcal{G}-\frac{Z_{J q}}{Z_{q q}} \mathcal{H},
\end{aligned}
$$

and similarly for $\mathcal{G}_{0}$ and $\mathcal{H}_{0}$. In summary, we find

$$
\left(\begin{array}{c}
\mathcal{F}_{0} \\
\mathcal{L}_{0} \\
\mathcal{H}_{0}
\end{array}\right)=\left(\begin{array}{ccc}
Z_{q q}^{-1} & -Z_{J q} Z_{q q}^{-1} & -Z_{J q} Z_{q q}^{-1} \\
0 & 1 & 0 \\
0 & 0 & 1
\end{array}\right)\left(\begin{array}{c}
\mathcal{F} \\
\mathcal{L} \\
\mathcal{H}
\end{array}\right) .
$$

From this matrix we can make several interesting observations. Firstly, we see that the operator $\frac{1}{4} F_{\mu v}^{2}(=\mathcal{F})$ indeed required the presence of the BRST exact operator $\mathcal{L}$ and of the gluon equation of motion operator $\mathcal{H}$ as these operators are "hidden" in the bare operator $\mathcal{F}$. Secondly, we do retrieve an upper triangular matrix, in agreement with the earlier description in (2.6). Moreover, we also find that the BRST exact operator $\mathcal{L}$ does not mix with $\mathcal{H}$, a mixing which is in principle allowed, but has a $Z$-factor equal to 1 . This can be nicely understood: the integrated BRST exact operator is in fact proportional to a sum of two (integrated) equations of motion terms,

$$
\int \mathrm{d}^{4} x\left(\partial_{\mu} b^{a} A_{\mu}^{a}+\partial_{\mu} \bar{c}^{a} D_{\mu}^{a b} c^{b}\right)=-\int \mathrm{d}^{4} x\left(b^{a} \partial_{\mu} A_{\mu}^{a}+\bar{c}^{a} \partial_{\mu} D_{\mu}^{a b} c^{b}\right)=-\int \mathrm{d}^{4} x\left(b^{a} \frac{\delta S}{\delta b^{a}}+\bar{c}^{a} \frac{\delta S}{\delta \bar{c}^{a}}\right),
$$

and therefore it does not mix with other operators, just like $\mathcal{H}$.

\section{The mixing matrix to all orders}

In this section, we shall demonstrate that we can determine the mixing matrix (2.44) exactly, i.e. to all orders of perturbation theory. For this purpose, we shall follow the lines of [40], suitably adapted to the gauge theory under study. We start with the following most general $(n+2 m+r)$-point function defined as,

$$
\begin{array}{r}
\mathcal{G}^{n+2 m+r}\left(x_{1}, \ldots, x_{n}, y_{1}, \ldots, y_{n}, \hat{y}_{1}, \ldots, \hat{y}_{n}, z_{1}, \ldots, z_{n}\right)=\left\langle A\left(x_{1}\right) \ldots A\left(x_{n}\right) c\left(y_{1}\right) \ldots c\left(y_{m}\right) \bar{c}\left(\hat{y}_{1}\right) \bar{c}\left(\hat{y}_{m}\right) b\left(z_{1}\right) \ldots b\left(z_{r}\right)\right\rangle \\
=\int[\mathrm{d} \phi] A\left(x_{1}\right) \ldots A\left(x_{n}\right) c\left(y_{1}\right) \ldots c\left(y_{m}\right) \bar{c}\left(\hat{y}_{1}\right) \bar{c}\left(\hat{y}_{m}\right) b\left(z_{1}\right) \ldots b\left(z_{r}\right) \mathrm{e}^{-S},(3.1)
\end{array}
$$

with the action $S$ given by

$$
S=S_{\mathrm{YM}}+S_{\mathrm{gf}}
$$


We have immediately assumed that there is an equal amount of ghost and antighost fields present as in any other case, the Green function (3.1) would be zero, due to ghost number symmetry. Subsequently, from the definition (3.1), we can immediately write down the connection between the renormalized Green function and the bare Green function,

$$
\mathcal{G}^{n+2 m+r}=Z_{A}^{-n / 2} Z_{c}^{-m} Z_{b}^{-r / 2} \mathcal{G}_{0}^{n+2 m+r} .
$$

From the previous equation, we shall be able to fix all the matrix elements of expression (2.44), based on the knowledge that

$$
\frac{\mathrm{d} \mathcal{G}^{n+2 m+r}}{\mathrm{~d} g^{2}}
$$

is finite.

We start by applying the chain rule when deriving the right hand side of equation 3.3 w.r.t. $g^{2}$. We find,

$$
\frac{\partial \mathcal{G}^{n^{\prime}}}{\partial g^{2}}=\frac{\partial g_{0}^{2}}{\partial g^{2}} \frac{\partial \mathcal{G}_{0}^{n^{\prime}}}{\partial g_{0}^{2}} Z_{A}^{-n / 2} Z_{c}^{-m} Z_{b}^{-r / 2}+\frac{\partial Z_{A}^{-n / 2}}{\partial g^{2}} Z_{c}^{-m} Z_{b}^{-r / 2} \mathcal{G}_{0}^{n^{\prime}}+Z_{A}^{-n / 2} \frac{\partial Z_{c}^{-m}}{\partial g^{2}} Z_{b}^{-r / 2} \mathcal{G}_{0}^{n^{\prime}}+Z_{A}^{-n / 2} Z_{c}^{-m} \frac{\partial Z_{b}^{-r / 2}}{\partial g^{2}} \mathcal{G}_{0}^{n^{\prime}},
$$

where we have replaced $(n+2 m+r)$ with $n^{\prime}$ as a shorthand. Next, we have to calculate all the derivatives w.r.t. $g^{2}$.

\section{- Calculation of $\frac{\partial g_{0}^{2}}{\partial g^{2}}$}

In dimensional regularization, with $d=4-\varepsilon$, one can write down

$$
g_{0}^{2}=\mu^{\varepsilon} Z_{g}^{2} g^{2} .
$$

Hence, if we derive this equation w.r.t. $g^{2}$,

$$
\frac{\partial g_{0}^{2}}{\partial g^{2}}=\mu^{\varepsilon} \frac{\partial Z_{g}^{2}}{\partial g^{2}} g^{2}+\mu^{\varepsilon} Z_{g}^{2}=g_{0}^{2}\left(\frac{\partial \ln Z_{g}^{2}}{\partial g^{2}}+\frac{1}{g^{2}}\right) .
$$

From the previous equation, we still have to determine $\frac{\partial \ln Z_{g}^{2}}{\partial g^{2}}$, which can be extracted from equation 3.6 . Deriving this equation w.r.t. $\mu$ gives,

$$
\mu \frac{\partial g_{0}^{2}}{\partial \mu}=\varepsilon \mu^{\varepsilon} Z_{g}^{2} g^{2}+\mu^{\varepsilon} \frac{\partial Z_{g}^{2}}{\partial g^{2}} \mu \frac{\partial g^{2}}{\partial \mu} g^{2}+\mu^{\varepsilon} Z_{g}^{2} \mu \frac{\partial g^{2}}{\partial \mu}=0
$$

were we have applied the chain rule again. We can rewrite this equation making use of the definition of the $\beta$-funtion

$$
\mu \frac{\partial g^{2}}{\partial \mu}=-\varepsilon g^{2}+\beta\left(g^{2}\right)
$$

where we have immediately extracted the part in $\varepsilon$, and we obtain,

$$
\frac{\partial \ln Z_{g}^{2}}{\partial g^{2}}=\frac{1}{g^{2}}\left(\frac{-\varepsilon g^{2}}{\mu \frac{\partial g^{2}}{\partial \mu}}-1\right)=\frac{1}{g^{2}}\left(\frac{-\beta\left(g^{2}\right)}{-\varepsilon g^{2}+\beta\left(g^{2}\right)}\right) .
$$

If we insert this result into expression (3.7), we ultimately find

$$
\frac{\partial g_{0}^{2}}{\partial g^{2}}=\frac{-\varepsilon g_{0}^{2}}{-\varepsilon g^{2}+\beta\left(g^{2}\right)} .
$$

- Calculation of $\frac{\partial Z_{A}^{-n / 2}}{\partial g^{2}}$

The next derivative w.r.t. $g^{2}$ can be calculated in a similar way. We start by applying the chain rule,

$$
\frac{\partial Z_{A}^{-n / 2}}{\partial g^{2}}=-n \frac{Z_{A}^{-n / 2}}{Z_{A}^{1 / 2}} \frac{\partial Z_{A}^{1 / 2}}{\partial g^{2}}=-n Z_{A}^{-n / 2} \frac{\partial \ln Z_{A}^{1 / 2}}{\partial g^{2}} .
$$


Next, we derive $\frac{\partial \ln Z_{A}^{1 / 2}}{\partial g^{2}}$ from the definition of the gluon anomalous dimension,

$$
\gamma_{A}=\mu \frac{\partial \ln Z_{A}^{1 / 2}}{\partial \mu}=\mu \frac{\partial g^{2}}{\partial \mu} \frac{\partial \ln Z_{A}^{1 / 2}}{\partial g^{2}}=\left(-\varepsilon g^{2}+\beta\left(g^{2}\right)\right) \frac{\partial \ln Z_{A}^{1 / 2}}{\partial g^{2}} .
$$

From expression (3.12) and (3.13), it now follows

$$
\frac{\partial Z_{A}^{-n / 2}}{\partial g^{2}}=-n Z_{A}^{-n / 2} \frac{\gamma_{A}}{-\varepsilon g^{2}+\beta\left(g^{2}\right)} .
$$

\section{- Calculation of $\frac{\partial Z_{c}^{-m}}{\partial g^{2}}$}

Completely analogously, we find with the help of the anomalous dimension of the ghost field,

$$
\begin{gathered}
\mu \frac{\partial Z_{c}^{1 / 2}}{\partial \mu}=\gamma_{c} Z_{c}^{1 / 2} \\
\frac{\partial Z_{c}^{-m}}{\partial g^{2}}=-2 m Z_{A}^{-n / 2} \frac{\gamma_{A}}{-\varepsilon g^{2}+\beta\left(g^{2}\right)} .
\end{gathered}
$$

- Calculation of $\frac{\partial Z_{b}^{-r / 2}}{\partial g^{2}}$

Finally, from

$$
\mu \frac{\partial Z_{b}^{1 / 2}}{\partial \mu}=\gamma_{b} Z_{c}^{1 / 2}
$$

we deduce

$$
\frac{\partial Z_{b}^{-r / 2}}{\partial g^{2}}=-r Z_{A}^{-n / 2} \frac{\gamma_{A}}{-\varepsilon g^{2}+\beta\left(g^{2}\right)}
$$

Taking all the previous results into account, we can rewrite expression (3.5),

$$
\frac{\mathrm{d} \mathcal{G}^{n^{\prime}}}{\mathrm{d} g^{2}}=\frac{Z_{A}^{-n / 2} Z_{c}^{-m} Z_{b}^{-r / 2}}{-\varepsilon g^{2}+\beta}\left[-\varepsilon g_{0}^{2} \frac{\partial}{\partial g_{0}^{2}}-n \gamma_{A}-2 m \gamma_{c}-r \gamma_{b}\right] \mathcal{G}_{0}^{n^{\prime}} .
$$

The right hand side of (3.19) still contains bare quantities, which we have to rewrite in terms of renormalized quantities. Notice also that we would like to get rid of the field numbers $n, m$ and $r$ as the mixing matrix will evidently will be independent of these numbers as they are arbitrary.

We shall now alter the right hand side of equation $(3.19)$ by calculating $\frac{\partial}{\partial g_{0}^{2}} \mathcal{G}_{0}^{n^{\prime}}$ and by removing the fields numbers. After a little bit of algebra, we obtain,

$$
\frac{\partial\left(\mathrm{e}^{-S}\right)}{\partial g_{0}^{2}}=-\int \mathrm{d}^{4} y\left(-\frac{1}{g_{0}^{2}}\left[\frac{F_{0}^{2}(y)}{4}\right]+\frac{1}{2 g_{0}^{2}}\left[A_{0}(y) \frac{\delta S}{\delta A_{0}(y)}\right]-\frac{1}{2 g_{0}^{2}}\left[b_{0}(y) \partial A_{0}(y)\right]\right) \mathrm{e}^{-S} .
$$

Consequently, deriving the $n^{\prime}$ point Green function $\mathcal{G}_{0}^{n^{\prime}}$ w.r.t. $g_{0}^{2}$ will result in several insertions in this Green function,

$$
g_{0}^{2} \frac{d \mathcal{G}_{0}^{n^{\prime}}}{d g_{0}}=\int \mathrm{d}^{4} y\left(\mathcal{G}_{0}^{n^{\prime}}\left\{\frac{F_{0}^{2}(y)}{4}\right\}-\frac{1}{2} \mathcal{G}_{0}^{n^{\prime}}\left\{A_{0}(y) \frac{\delta S}{\delta A_{0}(y)}\right\}+\frac{1}{2} \mathcal{G}_{0}^{n^{\prime}}\left\{b_{0}(y) \partial A_{0}(y)\right\}\right),
$$

where we have introduced a shorthand notation, e.g.

$$
\mathcal{G}_{0}^{n^{\prime}}\left\{\frac{F_{0}^{2}(y)}{4}\right\}=\left\langle\frac{F_{0}^{2}(y)}{4} A\left(x_{1}\right) \ldots A\left(x_{n}\right) c\left(y_{1}\right) \ldots c\left(y_{m}\right) \bar{c}\left(\hat{y}_{1}\right) \bar{c}\left(\hat{y}_{m}\right) b\left(z_{1}\right) \ldots b\left(z_{r}\right)\right\rangle .
$$


The field numbers can be rewritten by inserting the corresponding counting operator. If we start by inserting the counting operator for the gluon fields $n$, we find

$$
\int \mathrm{d}^{4} y \mathcal{G}_{0}^{n^{\prime}}\left\{A_{0}(y) \frac{\delta S}{\delta A_{0}(y)}\right\}=n \mathcal{G}_{0}^{n^{\prime}}
$$

as derived in equation (2.8). We can derive analogous relations for the other counting operators,

$$
\begin{aligned}
\int \mathrm{d}^{4} y \mathcal{G}_{0}^{n^{\prime}}\left\{c_{0}(y) \frac{\delta S}{\delta c_{0}(y)}\right\} & =m \mathcal{G}_{0}^{n^{\prime}} \\
\int \mathrm{d}^{4} y \mathcal{G}_{0}^{n^{\prime}}\left\{b_{0}(y) \frac{\delta S}{\delta b_{0}(y)}\right\} & =r \mathcal{G}_{0}^{n^{\prime}} .
\end{aligned}
$$

Taking all these results together, expression 3.19 now becomes,

$$
\begin{aligned}
\frac{d \mathcal{G}^{n^{\prime}}}{d g^{2}}= & \frac{Z_{A}^{-n / 2} Z_{c}^{-m} Z_{b}^{-r / 2}}{-\varepsilon g^{2}+\beta\left(g^{2}\right)} \int \mathrm{d}^{4} y\left[-\varepsilon\left(\mathcal{G}_{0}^{n^{\prime}}\left\{\mathcal{F}_{0}(y)\right\}-\frac{1}{2} \mathcal{G}_{0}^{n^{\prime}}\left\{\mathcal{H}_{0}(y)\right\}+\frac{1}{2} \mathcal{G}_{0}^{n^{\prime}}\left\{I_{0}(y)\right\}\right)\right. \\
& \left.-\gamma_{A}\left(g^{2}\right) \mathcal{G}_{0}^{n^{\prime}}\left\{\mathcal{H}_{0}(y)\right\}-2 \gamma_{c}\left(g^{2}\right) \mathcal{G}_{0}^{n^{\prime}}\left\{\mathcal{K}_{0}(y)\right\}-\gamma_{b}\left(g^{2}\right) \mathcal{G}_{0}^{n^{\prime}}\left\{I_{0}(y)\right\}\right] .
\end{aligned}
$$

We have again introduced a notational shorthand for the equation of motion operators, with $I_{0}=b_{0} \frac{\delta S}{\delta b_{0}}$ and $K_{0}=c_{0} \frac{\delta S}{\delta c_{0}}$ and with $\mathcal{F}$ and $\mathcal{H}$ already defined before.

In the last part of the manipulation of the $n^{\prime}$-point Green function we reexpress all the operators again in terms of their renormalized counterparts, thereby writing all the divergences explicitly in terms of $\varepsilon$. Firstly, we can reabsorb the $Z$-factors into $\mathcal{G}_{0}^{n^{\prime}}$ to find,

$$
\begin{aligned}
\frac{d \mathcal{G}^{n^{\prime}}}{d g^{2}}= & \frac{1}{-\varepsilon g^{2}+\beta\left(g^{2}\right)} \int \mathrm{d}^{4} y\left[-\varepsilon\left(\mathcal{G}^{n^{\prime}}\left\{\mathcal{F}_{0}(y)\right\}-\frac{1}{2} \mathcal{G}^{n^{\prime}}\left\{\mathcal{H}_{0}(y)\right\}+\frac{1}{2} \mathcal{G}_{0}^{n^{\prime}}\left\{I_{0}(y)\right\}\right)\right. \\
& \left.-\gamma_{A}\left(g^{2}\right) \mathcal{G}^{n^{\prime}}\left\{\mathcal{H}_{0}(y)\right\}-2 \gamma_{c}\left(g^{2}\right) \mathcal{G}^{n^{\prime}}\left\{\mathcal{K}_{0}(y)\right\}-\gamma_{b}\left(g^{2}\right) \mathcal{G}^{n^{\prime}}\left\{I_{0}(y)\right\}\right] .
\end{aligned}
$$

Secondly, we parametrize the mixing matrix (2.44),

$$
\left(\begin{array}{l}
\mathcal{F}_{0} \\
\mathcal{L}_{0} \\
\mathcal{H}_{0}
\end{array}\right)=\left(\begin{array}{ccc}
1+\frac{a}{\varepsilon} & -\frac{b}{\varepsilon} & -\frac{b}{\varepsilon} \\
0 & 1 & 0 \\
0 & 0 & 1
\end{array}\right)\left(\begin{array}{c}
\mathcal{F} \\
\mathcal{L} \\
\mathcal{H}
\end{array}\right) .
$$

Here we have displayed the fact that the entries associated with $a\left(g^{2}, \varepsilon\right)$ and $b\left(g^{2}, \varepsilon\right)$, which represent a formal power series in $g^{2}$, must at least have a simple pole in $\varepsilon$. We recall that the integrated operator $\mathcal{L}_{0}$ is proportional to the sum of the two counting operators $\int I_{0}$ and $\int \mathcal{K}_{0}$, see expression (2.45). Therefore $\int I_{0}=\int I$ and $\int \mathcal{K}_{0}=\int \mathcal{K}$. Inserting all this information into expression $(3.26$ yields,

$$
\begin{array}{r}
\frac{\partial \mathcal{G}^{n^{\prime}}}{\partial g^{2}}=\frac{1}{-\varepsilon g^{2}+\beta\left(g^{2}\right)} \int \mathrm{d}^{4} y\left[\mathcal { G } ^ { n ^ { \prime } } \left\{(-\varepsilon-a) \mathcal{F}(y)-b I(y)-b \mathcal{K}(y)+b \mathcal{H}(y)+\frac{\varepsilon}{2} \mathcal{H}(y)-\frac{\varepsilon}{2} I(y)\right.\right. \\
\left.\left.-\gamma_{A}\left(g^{2}\right) \mathcal{H}(y)-2 \gamma_{c}\left(g^{2}\right) \mathcal{K}(y)-\gamma_{b}\left(g^{2}\right) I(y)\right\}\right],
\end{array}
$$

giving us the final result from which we shall be able to fix the matrix elements of expression (2.44).

As the left hand side of our final expression $(3.28)$ is finite, the right hand side is finite too. Therefore, the following 
coefficients must be finite,

$$
\begin{aligned}
\mathcal{F} & : \quad \frac{-\varepsilon-a}{-\varepsilon g^{2}+\beta\left(g^{2}\right)}=\frac{1}{g^{2}} \frac{(1+a / \varepsilon)}{1-\beta\left(g^{2}\right) /\left(\varepsilon g^{2}\right)}, \\
I & : \quad \frac{-\varepsilon / 2-b-\gamma_{b}\left(g^{2}\right)}{-\varepsilon g^{2}+\beta\left(g^{2}\right)}=\frac{1}{2 g^{2}} \frac{1+2\left(b+\gamma_{b}\left(g^{2}\right)\right) / \varepsilon}{1-\beta\left(g^{2}\right) /\left(\varepsilon g^{2}\right)}, \\
\mathcal{H} & : \frac{\varepsilon / 2+b-\gamma_{A}\left(g^{2}\right)}{-\varepsilon g^{2}+\beta\left(g^{2}\right)}=-\frac{1}{2 g^{2}} \frac{1+2\left(b-\gamma_{A}\left(g^{2}\right)\right) / \varepsilon}{1-\beta\left(g^{2}\right) /\left(\varepsilon g^{2}\right)}, \\
\mathcal{K} & : \quad \frac{-b-2 \gamma_{c}\left(g^{2}\right)}{-\varepsilon g^{2}+\beta\left(g^{2}\right)},
\end{aligned}
$$

seen as a power series in $g^{2}$. This can only be true if

$$
\begin{aligned}
& a\left(g^{2}, \varepsilon\right)=-\frac{\beta\left(g^{2}\right)}{g^{2}}, \\
& b\left(g^{2}, \varepsilon\right)=\gamma_{A}\left(g^{2}\right)-\frac{1}{2} \frac{\beta\left(g^{2}\right)}{g^{2}}=-\gamma_{b}\left(g^{2}\right)-\frac{1}{2} \frac{\beta\left(g^{2}\right)}{g^{2}}=-2 \gamma_{c}\left(g^{2}\right) .
\end{aligned}
$$

In fact, this last equation reveals a connection between the anomalous dimension of $A$ and $b$, and between the anomalous dimension of $A, g$ and $c$, namely

$$
\begin{aligned}
\gamma_{A}+\gamma_{b} & =0, \\
\gamma_{A}+2 \gamma_{c} & =\frac{\beta}{2 g^{2}} .
\end{aligned}
$$

These relations are well-known to hold in the Landau gauge, since $Z_{A} Z_{b}=1$ and $Z_{c} Z_{A}^{1 / 2} Z_{g}=1$, as derived from the algebraic renormalization analysis, which leads to equations (2.38) and (2.39).

In summary, we have completely fixed the mixing matrix in term of the elementary renormalization group functions, and this to all orders of perturbation theory,

$$
\underbrace{\left(\begin{array}{c}
\mathcal{F}_{0} \\
\mathcal{L}_{0} \\
\mathcal{H}_{0}
\end{array}\right)}_{X_{0}}=\underbrace{\left(\begin{array}{ccc}
1-\frac{\beta\left(g^{2}\right) / g^{2}}{\varepsilon} & -\frac{2 \gamma_{c}\left(g^{2}\right)}{\varepsilon} & -\frac{2 \gamma_{c}\left(g^{2}\right)}{\varepsilon} \\
0 & 1 & 0 \\
0 & 0 & 1
\end{array}\right)}_{Z} \underbrace{\left(\begin{array}{c}
\mathcal{F} \\
\mathcal{L} \\
\mathcal{H}
\end{array}\right)}_{X} .
$$

In addition, as a check of this result, we have also uncovered two relations, 3.31, between anomalous dimensions which must hold for consistency. These correspond to (2.38) and (2.39), which are well-known nonrenormalization theorems in the Landau gauge.

\section{Constructing a renormalization group invariant}

As a last step, we can now look for a renormalization group invariant operator by determining the anomalous dimension $\Gamma$ coming from the mixing matrix $Z$. We define the anomalous dimension matrix $\Gamma$ as

$$
\mu \frac{\partial}{\partial \mu} Z=Z \Gamma \text {. }
$$

For the calculation of $\Gamma$, we require

$$
\begin{aligned}
\mu \frac{\partial}{\partial \mu}\left(1-\frac{\beta / g^{2}}{\varepsilon}\right) & =\frac{1}{\varepsilon}\left(-\varepsilon g^{2}+\beta\left(g^{2}\right)\right) \frac{\partial\left(\beta / g^{2}\right)}{\partial g^{2}} \\
-\mu \frac{\partial}{\partial \mu} \frac{\gamma_{c}}{\varepsilon} & =\frac{1}{\varepsilon}\left(-\varepsilon g^{2}+\beta\left(g^{2}\right)\right) \frac{\partial \gamma_{c}}{\partial g^{2}},
\end{aligned}
$$

so we obtain,

$$
\Gamma=\left(\begin{array}{ccc}
g^{2} \frac{\partial\left(\beta / g^{2}\right)}{\partial g^{2}} & -g^{2} \frac{\partial \gamma_{c}}{\partial g^{2}} & -g^{2} \frac{\partial \gamma_{c}}{\partial g^{2}} \\
0 & 0 & 0 \\
0 & 0 & 0
\end{array}\right)
$$


which is indeed finite, a nice consistency check. This matrix is then related to the anomalous dimension of the operators:

$$
\begin{aligned}
X_{0}=Z X & \Rightarrow 0=\mu \frac{\partial Z}{\partial \mu} X+Z \mu \frac{\partial X}{\partial \mu} \\
& \Rightarrow \mu \frac{\partial X}{\partial \mu}=-\Gamma X .
\end{aligned}
$$

We now have all the ingredients at our disposal to determine a renormalization group invariant operator. We are looking for a linear combination of $\mathcal{F}, \mathcal{L}$ and $\mathcal{H}$ which does not run,

$$
\mu \frac{\partial}{\partial \mu}[k \mathcal{F}+\ell \mathcal{G}+m \mathcal{H}]=0
$$

whereby $k, \ell$ and $m$ are to be understood as functions of $g^{2}$. Invoking the chain rule gives

$$
\mu \frac{\partial k}{\partial \mu} \mathcal{F}-k g^{2} \frac{\partial\left(\beta / g^{2}\right)}{\partial g^{2}} \mathcal{F}+k g^{2} \frac{\partial \gamma_{c}}{\partial g^{2}} \mathcal{L}+k g^{2} \frac{\partial \gamma_{c}}{\partial g^{2}} \mathcal{H}+\mu \frac{\partial \ell}{\partial \mu} \mathcal{L}+\mu \frac{\partial m}{\partial \mu} \mathcal{H}=0 .
$$

This previous equation results in two differential equations,

$$
\left\{\begin{array}{l}
\mu \frac{\partial k}{\partial \mu}-k g^{2} \frac{\partial\left(\beta / g^{2}\right)}{\partial g^{2}}=0 \\
\mu \frac{\partial \ell}{\partial \mu}+k g^{2} \frac{\gamma_{c}}{\partial g^{2}}=0 \\
\ell=m
\end{array}\right.
$$

which can be solved by,

$$
\left\{\begin{array}{l}
k\left(g^{2}\right)=\frac{\beta\left(g^{2}\right)}{g^{2}}, \\
\ell\left(g^{2}\right)=m\left(g^{2}\right)=-\gamma_{c}\left(g^{2}\right) .
\end{array}\right.
$$

In summary, we have determined a renormalization group invariant scalar operator $\mathcal{R}$ containing $\mathcal{F}$. Explicitly,

$$
\mathcal{R}=\frac{1}{4} \frac{\beta\left(g^{2}\right)}{g^{2}} F_{\mu \nu}^{2}-\gamma_{c}\left(g^{2}\right)\left(A_{\mu}^{a} \partial_{\mu} b^{a}+\partial_{\mu} \bar{c}^{a} D_{\mu}^{a b} c^{b}\right)-\gamma_{c}\left(g^{2}\right) A_{\mu}^{a} \frac{\delta S}{\delta A_{\mu}^{a}},
$$

without having calculated any loop diagram. Moreover, this invariant is equal to the trace anomaly $\Theta_{\mu}^{\mu}$, which is expected as $\Theta_{\mu}^{\mu}$ is also a $d=4$ renormalization group invariant [37].

\section{Conclusion}

In this paper, we have provided a detailed analysis of the renormalization of the non-integrated operator $F_{\mu v}^{2}$ in Yang-Mills gauge theory in the Landau gauge, and this to all orders. We have shown that this operator mixes with two other operators: the BRST exact operator $s\left(\bar{c}^{a} \partial_{\mu} A_{\mu}^{a}\right)$ and a gluon equation of motion operator, $A_{\mu}^{a} \frac{\delta S}{\delta A_{\mu}^{a}}$. We have composed the corresponding renormalization matrix $Z$ and we have been able to determine this matrix to all orders in perturbation theory. Several checks have confirmed the results. We have recovered the well known non-renormalization theorems in the Landau gauge, relating the gluon, ghost, auxiliary field and coupling constant anomalous dimension. In addition, we have calculated the anomalous dimension matrix using $Z$, which was nicely finite, and we have been able to construct a renormalization group invariant containing $F_{\mu v}^{2}$.

When turning to physical states, the BRST exact term and the equation of motion term will drop and be no longer relevant. However, we have paved the way for more complicated actions as this framework is very solid. For example, in the Gribov-Zwanziger action, when investigating the operator $F_{\mu v}^{2}$, we also expect a mixing. Again, we would expect three different classes of operators, the gauge invariant, the BRST exact and the ones which vanish upon using the equations of motion. However, the Gribov-Zwanziger action is no longer BRST invariant [27, 41]. Fortunately, the framework we have set up can be saved as one can embed the Gribov-Zwanziger action into a "larger" BRST invariant action which will reduce to the known Gribov-Zwanziger action in the so-called 
physical limit [27]. The embedding into a BRST invariant action seems to be the crucial tool to correctly identify the relevant, renormalizable, gauge invariant operators in the Gribov-Zwanziger case, as the physical limit of their more general counterparts constructed from the larger action. In this case, the BRST exact operators shall no longer be automatically irrelevant when taking the physical limit, as the BRST symmetry is softly broken by the restriction to the Gribov region. Therefore, we expect a non-trivial mixing to occur when we transfer to the physical reality of glueballs, i.e. when calculating the corresponding correlator. Hence, it will certainly be of the utmost importance to take all the possible mixings into account in order to be able to construct a renormalization group invariant [42].

We did not include fermions in our analysis. The cohomological treatment of massless fermions is not that complicated, see [39]. However, additional equation of motion terms, as well as the derivative of the singlet vector current can be relevant. In addition, including non-degenerate fermion masses would complicate matters, due to the introduction of a fermion mass mixing matrix. As we already mentioned, our interest is and will be mainly focused on pure gauge theories [42].

\section{Acknowledgments.}

We wish to thank J. A. Gracey for many helpful comments. D. Dudal and N. Vandersickel are supported by the Research Foundation - Flanders (FWO). The Conselho Nacional de Desenvolvimento Científico e Tecnológico (CNPq-Brazil), the Faperj, Fundação de Amparo à Pesquisa do Estado do Rio de Janeiro, the SR2-UERJ and the Coordenação de Aperfeiçoamento de Pessoal de Nível Superior (CAPES) are gratefully acknowledged for financial support. This work is supported in part by funds provided by the US Department of Energy (DOE) under cooperative research agreement DEFG02-05ER41360. N. Vandersickel acknowledges the hospitality at the CTP (MIT) and UERJ, and D. Dudal that at the UERJ, where parts of this work were done.

\section{References}

[1] V. Mathieu, N. Kochelev and V. Vento, arXiv:0810.4453 [hep-ph].

[2] D. Bettoni, J. Phys. Conf. Ser. 9 (2005) 309.

[3] M. S. Chanowitz, Int. J. Mod. Phys. A 21 (2006) 5535.

[4] D. S. Carman, AIP Conf. Proc. 814 (2006) 173.

[5] B. Alessandro et al. [ALICE Collaboration], J. Phys. G 32 (2006) 1295.

[6] C. J. Morningstar and M. J. Peardon, Phys. Rev. D 60 (1999) 034509.

[7] C. McNeile, Nucl. Phys. A 711 (2002) 303.

[8] Y. Chen et al., Phys. Rev. D 73 (2006) 014516.

[9] M. J. Teper, arXiv:hep-th/9812187.

[10] H. B. Meyer, arXiv:0808.3151 [hep-lat].

[11] G. B. West, Nucl. Phys. Proc. Suppl. 54A (1997) 353.

[12] R. L. Jaffe and K. Johnson, Phys. Lett. B 60 (1976) 201.

[13] J. M. Cornwall, Phys. Rev. D 26 (1982) 1453.

[14] C. W. Bernard, Phys. Lett. B 108 (1982) 431.

[15] J. M. Cornwall and A. Soni, Phys. Lett. B 120 (1983) 431.

[16] T. Barnes, Z. Phys. C 10 (1981) 275.

[17] A. Szczepaniak, E. S. Swanson, C. R. Ji and S. R. Cotanch, Phys. Rev. Lett. 76 (1996) 2011.

[18] A. B. Kaidalov and Yu. A. Simonov, Phys. Atom. Nucl. 63 (2000) 1428 [Yad. Fiz. 63 (2000) 1428].

[19] V. A. Novikov, M. A. Shifman, A. I. Vainshtein and V. I. Zakharov, Nucl. Phys. B 165 (1980) 67. 
[20] S. Narison, arXiv:0811.0563 [hep-ph].

[21] E. V. Shuryak, Nucl. Phys. B 203 (1982) 116.

[22] R. C. Brower, S. D. Mathur and C. I. Tan, Nucl. Phys. B 587 (2000) 249.

[23] H. Forkel, Phys. Rev. D 78 (2008) 025001.

[24] D. Zwanziger, Nucl. Phys. B 323 (1989) 513.

[25] V. N. Gribov, Nucl. Phys. B 139 (1978) 1.

[26] D. Dudal, S. P. Sorella, N. Vandersickel and H. Verschelde, Phys. Rev. D 77 (2008) 071501.

[27] D. Dudal, J. A. Gracey, S. P. Sorella, N. Vandersickel and H. Verschelde, Phys. Rev. D 78 (2008) 065047.

[28] J. C. Collins, Phys. Rev. D 14 (1976) 1965.

[29] H. Kluberg-Stern and J. B. Zuber, Phys. Rev. D 12 (1975) 467.

[30] J. C. Collins, Renormalization. An Introduction To Renormalization, The Renormalization Group, And The Operator Product Expansion, Cambridge, Uk: Univ. Pr. (1984).

[31] J. A. Gracey, Nucl. Phys. B 634 (2002) 192 [Erratum-ibid. B 696 (2004) 295].

[32] S. D. Joglekar and B. W. Lee, Annals Phys. 97 (1976) 160.

[33] W. S. Deans and J. A. Dixon, Phys. Rev. D 18 (1978) 1113.

[34] D. Espriu, Phys. Rev. D 28 (1983) 349.

[35] M. Henneaux, Phys. Lett. B 313 (1993) 35 [Erratum-ibid. B 316 (1993) 633].

[36] J. C. Collins and R. J. Scalise, Phys. Rev. D 50 (1994) 4117.

[37] J. C. Collins, A. Duncan and S. D. Joglekar, Phys. Rev. D 16 (1977) 438.

[38] R. Tarrach, Nucl. Phys. B 196 (1982) 45.

[39] O. Piguet and S. P. Sorella, Lect. Notes Phys. M28 (1995) 1.

[40] L. S. Brown, Annals Phys. 126 (1980) 135.

[41] D. Zwanziger, Nucl. Phys. B 399 (1993) 477.

[42] D. Dudal, S. P. Sorella, N. Vandersickel and H. Verschelde, work in progress. 